\title{
Evaluation of three techniques for differential diagnosis of prostatic needle biopsy specimens
}

\author{
R F T McMahon, L J McWilliam, S Mosley
}

Abstract

Aims: To determine whether acidic mucin staining, lectin histochemistry using Wisteria floribunda agglutinin, and immunohistochemistry using the monoclonal antibody EAB 903 are of benefit in distinguishing between hyperplastic and carcinomatous prostatic glandular tissue in needle biopsy specimens.

Methods: Formalin fixed, paraffin wax embedded prostatic needle biopsy specimens of benign and malignant tissue were examined. Alcian blue-periodic acid Schiff staining was performed on 33 benign and 34 malignant cases. Wisteria floribunda agglutinin (WFA) binding sites were demonstrated by the avidin-biotin peroxidase (ABC) technique with and without neuraminidase pretreatment on 34 benign cases and 32 malignant cases. EAB903 anticytokeratin antibody binding sites were demonstrated using both an indirect immunoperoxidase (IIP) technique and an avidin-biotin peroxidase complex method on seven benign and 31 malignant cases.

Results: Acidic mucin staining was found in 17 of 34 malignant glands and was weakly positive in five of 33 benign glands.
WFA positivity before neuraminidase pretreatment was present in 29 of 32 malignant glands and in 19 of 34 benign glands. After neuraminidase all benign and malignant cases showed positivity. EAB 903 positivity was seen in 11 of 31 malignant glands using the IIP technique and in two of 31 malignant glands using the ABC technique. In seven benign cases there was positivity in all glands using the IIP method with predominant basal cell staining in three and superficial cell staining in four. In benign cases using the ABC method two cases were negative.

Conclusions: None of the three methods studied showed sufficient sensitivity and specificity to allow their recommendation for routine diagnostic use.

$(\mathcal{F}$ Clin Pathol 1992;45:1094-1098)

Needle biopsy, by the transrectal or perineal routes, is an increasingly popular method of diagnosis in prostatic practice. Relatively small amounts of tissue are available from this technique in comparison with transurethral resections or total prostatectomies, thus magnifying the degree of diagnostic difficulty.
Division of

Histopathology,

Department of

Pathological Sciences, University of

Manchester, Stopford

Building, Oxford

Road, Manchester

M13 9PT

R F T McMahon

$S$ Mosley

Withington Hospital, Manchester

L J McWilliam

Correspondence to:

Dr R F T McMahon

Accepted for publication 8 July 1992

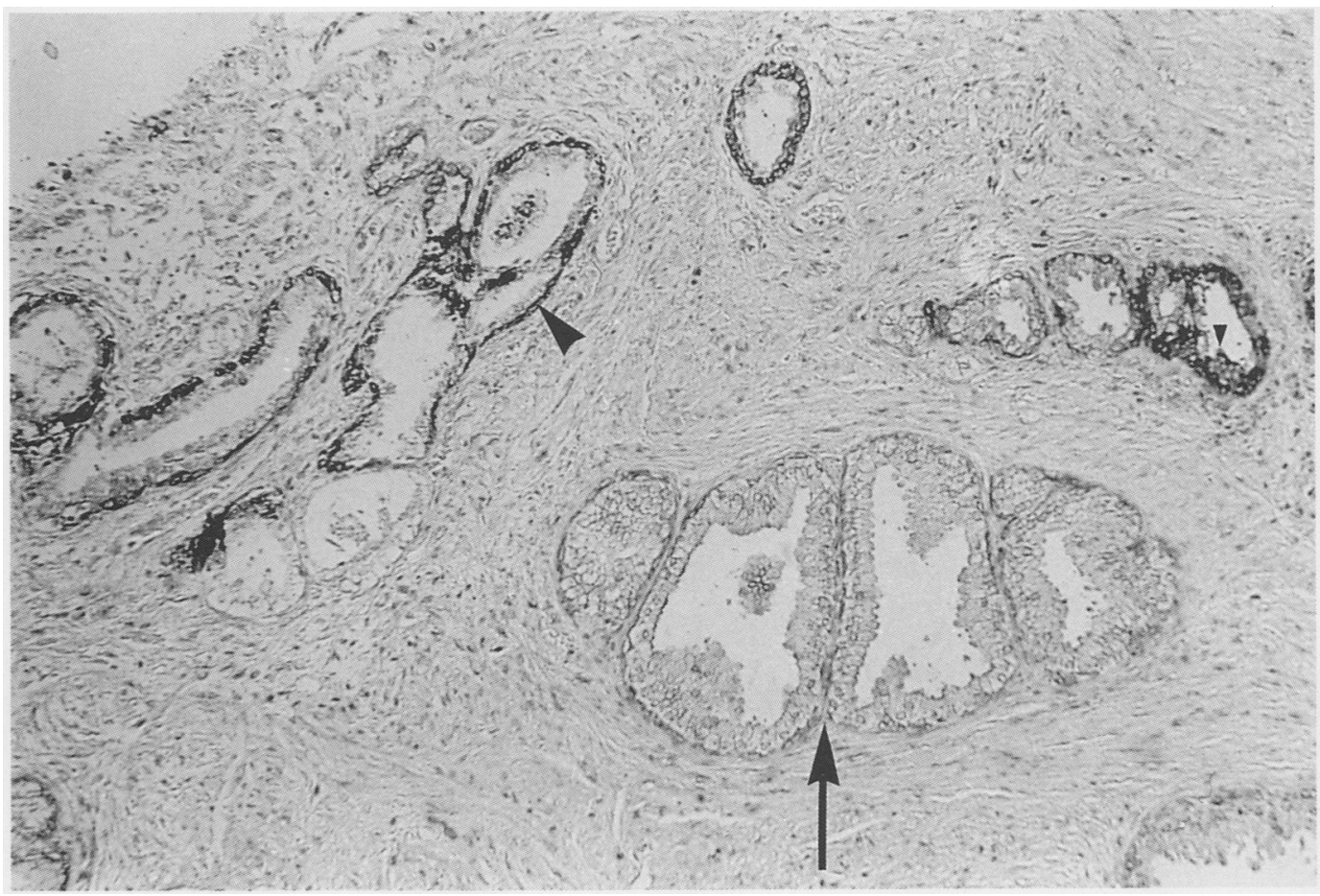

Figure 1 EAB 903 immunoperoxidase staining of benign prostatic glands using the IIP method. Note negative glands (arrow), basal cell staining in some glands (arrowhead), and superficial cell staining focally (small arrowhead). 
Figure 2 Focal positivity (arrow) of malignant prostate for $E A B 903$ using the IIP method.

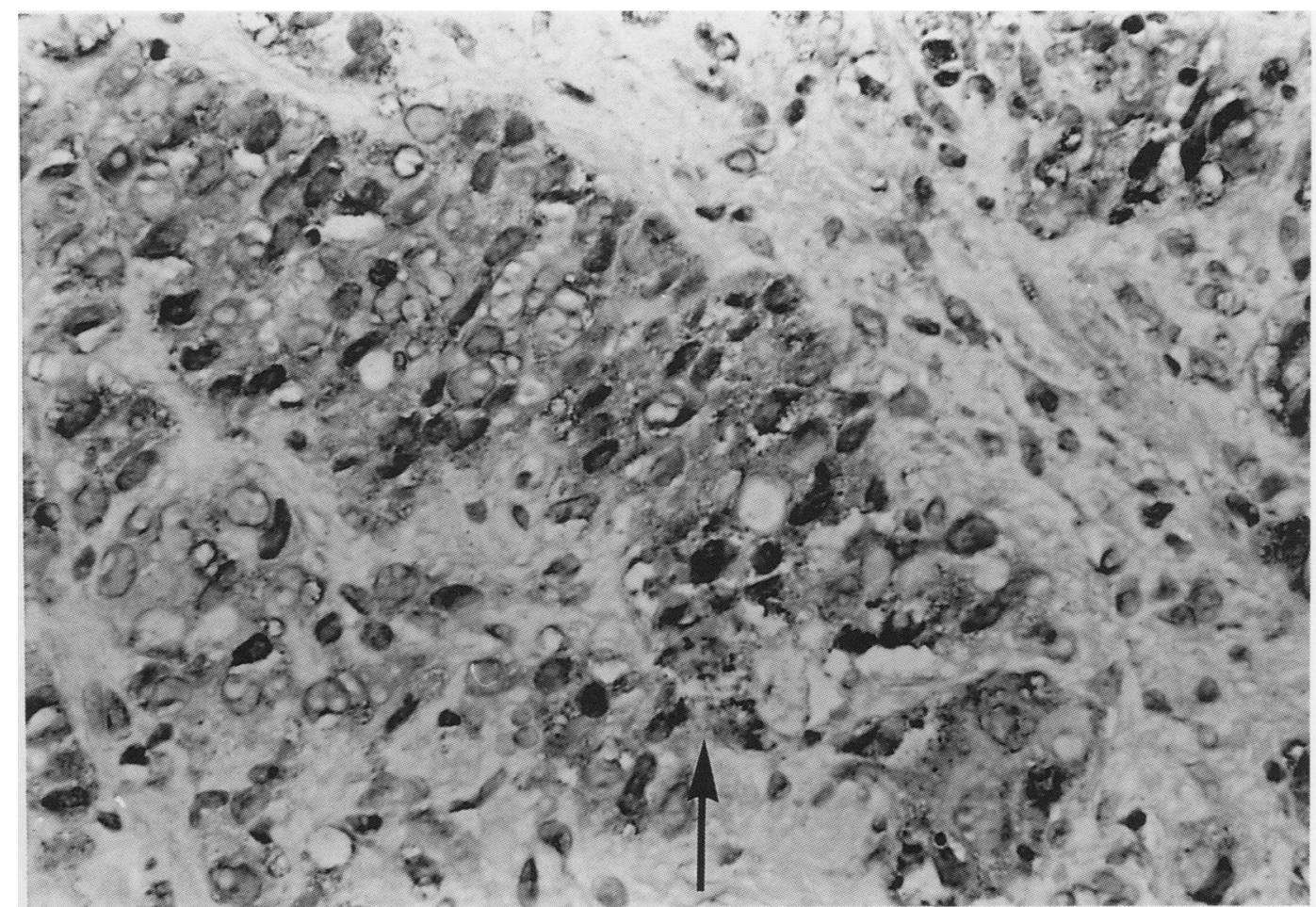

Various special techniques have been proposed to aid in the differential diagnosis of benign from malignant lesions. Carcinomas of the prostate secrete acidic mucin in $40-70 \%$ of cases, while normal and hyperplastic prostate glands produce only neutral mucin. ${ }^{1}$ In a preliminary study of lectin histochemistry, Wisteria floribunda agglutinin (WFA) seemed to be expressed only by malignant glands, probably related to sialylation, as benign glands contained binding sites for WFA after neuraminidase digestion. ${ }^{2} \mathrm{~A}$ monoclonal anticytokeratin antibody, EAB 903, has been shown by several groups to be exhibited only by the basal cells of benign prostatic glands, ${ }^{3-6}$ while these EAB 903 reactive cells are absent in malignant prostatic glands, with the exception of two reported cases. ${ }^{7}$ This study was undertaken to determine whether any or all of these techniques might be of benefit in distinguishing between hyperplastic and carcinomatous prostatic glandular tissue in needle biopsy specimens.

\section{Methods}

Needle biopsy specimens of prostate gland were obtained from the files of the Department of Surgical Histology, Manchester Royal Infirmary, and of the Department of Histopathology, Withington Hospital: 67 cases ( 33 benign, 34 malignant) were evaluated in the mucin studies, 66 cases were examined with WFA lectin ( 34 benign, 32 malignant) and 38 cases were studied with EAB 903 (seven benign, 31 malignant). The usual diagnostic criteria for differentiating benign from malignant prostatic tissue were applied. ${ }^{8}$ Of the 31 cases evaluated with the monoclonal antibody, in combination with mucin and lectin histochemical techniques, Gleason grading ${ }^{9}$ showed one grade 2, one grade $2+3,12$ grade 3 , four grade $3+4$, one grade $3+5$, eight grade 4 , two grade $4+5$ and two grade 5 tumours. The Brawn modification of the MD Anderson Hospital system ${ }^{10}$ disclosed five grade 1,16 grade 2 , and 10 grade 3 carcinomas.

All of the tissue had been fixed in neutral buffered $10 \%$ formalin and routinely processed, before embedding in paraffin wax. The sections were dewaxed and rehydrated. A combined alcian blue ( $\mathrm{pH} 2.5)$ and periodic acid Schiff stain after diastase pretreatment (AB-DPAS) was performed. Wisteria floribunda agglutinin binding sites were demonstrated by the avidin-biotin peroxidase technique using a lectin concentration of $10 \mu \mathrm{g} / \mathrm{ml}$; neuraminidase pretreatment to remove sialic acid residues was also performed. The antibody EAB 903, which was purchased from Enzo Diagnostics, is a high molecular weight $(68,58$, 56.5 and 56 kilodaltons) anticytokeratin monoclonal antibody ${ }^{11}$ and binding sites were identified by two methods. The first was a standard indirect immunoperoxidase technique. Endogenous peroxidase activity was blocked, sections were digested with trypsin, and the primary antibody was applied for 60 minutes at a dilution of 1 in 100. The secondary rabbit anti-mouse antibody was applied for 30 minutes, the reaction product was developed in diaminobenzidine (DAB), and the sections were counterstained with Harris's haematoxylin. The avidin-biotin peroxidase complex method was also used. Again endogenous peroxidase activity was blocked and sections were digested with trypsin. The primary antibody was applied at a dilution of 1 in 100 for 60 minutes, followed by the secondary sheep anti-mouse biotinylated antibody at a dilution of 1 in 100 for 30 minutes. Sections were then incubated in freshly made up avidin- 
Figure 3 Focal positivity (arrowheads) of malignant prostate for $E A B 903$ using the $A B C$ method.

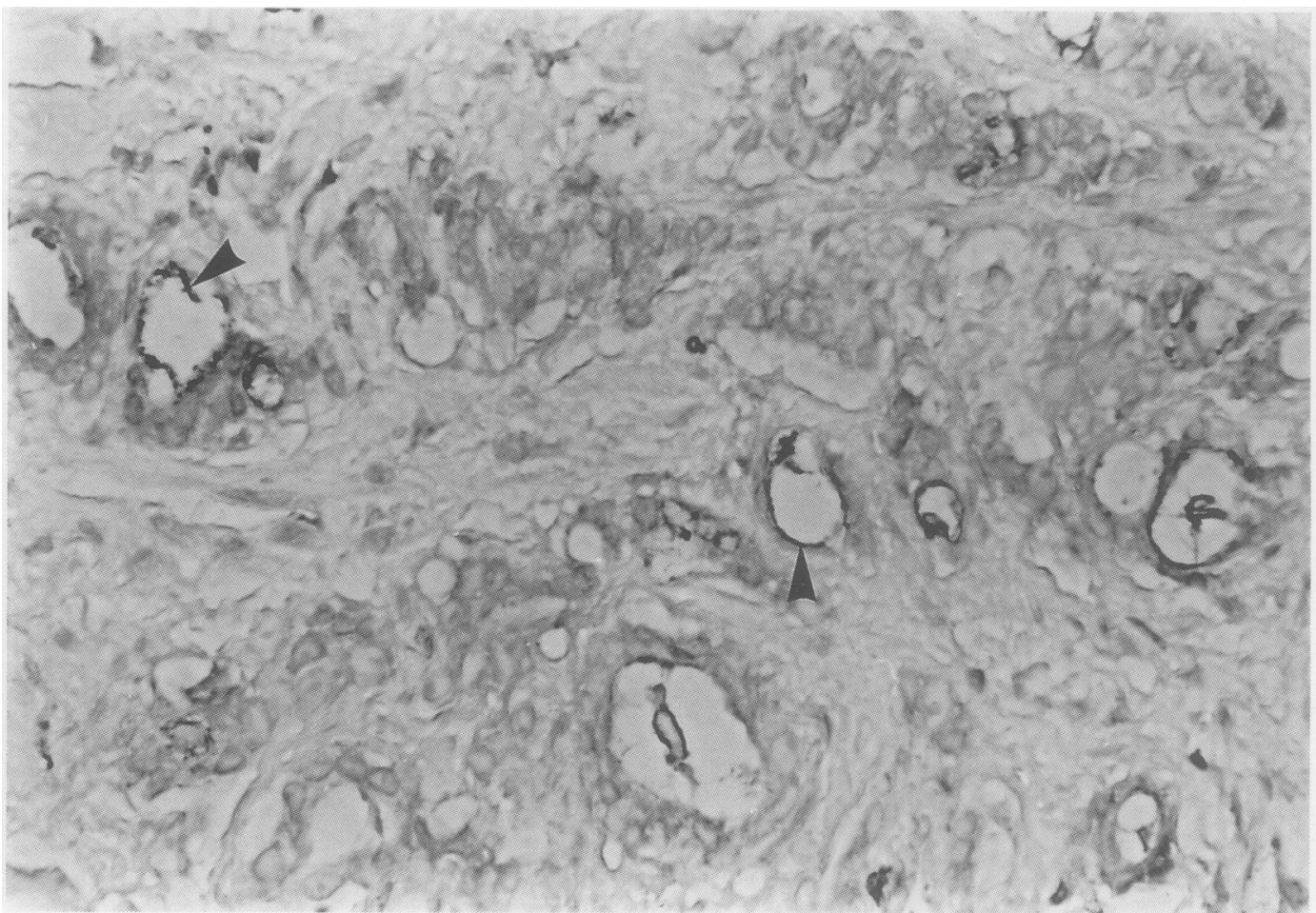

biotin-peroxidase complex for 60 minutes. The reaction product was developed in DAB and sections were counterstained with methyl green.

\section{Results}

The results of mucin staining are shown in table 1 . The sections were initially assessed for the presence of alcianophilia (blue staining) on an absent/definitely present/weakly positive basis. The last two grades were then placed into a "present" category. By this method, 50\% (17/34) of the malignant glands contained acidic mucin while $15 \%(5 / 33)$ of the benign glands stained positively for alcian blue, albeit only weakly so in all five cases.

The results of Wisteria lectin studies are shown in table 1. Again, a simplified absent/ present grading was used. Before neuraminidase pretreatment, $90 \%$ (29/32) of malignant prostates revealed binding sites for WFA while $56 \%$ (19/34) of benign glands did so, although 17 of these cases were only weakly positive. After neuraminidase, all glands were uniformly positive, both benign and malignant.

The findings with EAB 903 are shown in table 2. In benign glands all seven cases assessed by the IIP method were positive. Reactivity was noted in basal cells predominantly in three instances and in superficial/ luminal cells predominantly in four cases. With the ABC method, two of the seven cases were negative, three were only weakly positive, and only two showed definite basal cell reactivity. In malignant prostates 11 of the $31(36 \%)$ cases were reactive for EAB 903 by the IIP method and two of $31(6 \%)$ cases were positive by the ABC method.

Table 3 shows the results of comparing the combination of staining patterns with the Gleason grade and simplified histological dif- ferentiation in the 31 cases where all modalities were used. Gleason grading is extremely difficult to perform on needle biopsy material due to the paucity of malignant tissue available and an assessment of differentiation was easier to apply in this circumstance. There did not seem to be any significant differences in the distribution of either Gleason grades or degree of differentiation when compared with the use of these three techniques, including both modifications of the monoclonal antibody method, as a panel.

\section{Discussion}

Prostatic needle biopsy specimens are a challenge to the diagnostic histopathologist in that relatively small amounts of tissue are provided from which clinically important decisions may be made. To improve the accuracy of diagnosis, several methods have been used, including mucin histochemistry, ${ }^{12-15}$ lectin histochemistry, ${ }^{216}$ and immunohistochemistry. ${ }^{3-7} 1718$

Mucin histochemistry has been used for many years and while acidic mucin can be demonstrated in $40-70 \%$ of malignant cases, it is rarely seen in benign hyperplastic glands. In this study $50 \%$ of carcinomas contained alcianophilic mucin, an increase from the $38 \%$ of positive cases previously reported on transurethral resection material. ${ }^{1}$ In that series no benign glands exhibiting acidic mucin were

Table 1 Prostatic needle biopsy specimens

\begin{tabular}{llccl}
\hline & & Positive & Negative & Total \\
\hline Alcian blue & Benign & 5 & 28 & 33 \\
& Malignant & 17 & 17 & 34 \\
WFA-NA & Benign & 19 & 15 & 34 \\
& Malignant & 29 & 3 & 32 \\
WFA + NA & Benign & 34 & 0 & 34 \\
& Malignant & 32 & 0 & 32 \\
\hline
\end{tabular}


Table 2 Prostatic needle biopsy specimens: EAB 903 staining

\begin{tabular}{lllll}
\hline & Method & Negative & Positive B > S & Positive S > B \\
\hline Benign & IIP & 0 & 3 & 4 \\
& ABC & 2 & 2 & 3 \\
\multirow{4}{*}{ Malignant } & & Negative & Positive & \\
& IIP & 20 & 11 & \\
& ABC & 29 & 2 &
\end{tabular}

B > S: Basal greater than superficial staining

$\mathrm{S}>\mathrm{B}$ : Superficial more than basal staining

Table 3 Comparison of staining patterns with mucin, monoclonal antibody, and lectin histochemistry with Gleason grade and $M D A H$ grading systems

\begin{tabular}{llll}
\hline Staining pattern & No of cases & Gleason' $^{\prime}$ & MDAH $^{\prime \prime}$ \\
\hline AB +, IIP -, ABC -, WFA + & 7 & $2+3,3,3,3+4,4,4,5$ & $2,2,3,3,1,2,2$ \\
AB +, IIP -, ABC +, WFA + & 2 & $3,3+4$ & 2,2 \\
AB +, IIP +, ABC -, WFA - & 1 & 4 & 2 \\
AB +, IIP +, ABC -, WFA + & 7 & $3,3,3,3,3+4,4,5$ & $1,3,2,2,2,3,2$ \\
AB -, IIP -, ABC -, WFA - & 2 & $2,4+5$ & 1,3 \\
AB -, IIP -, ABC -, WFA + & 9 & $3,3,3,3,3,3+4,4,4,4+5$ & $2,1,2,3,2,1,3,2,2$ \\
AB -, IIP +, ABC -, WFA + & 3 & $3+5,4,4$ & $3,3,3$ \\
\hline Staining: & \\
AB: Alcian blue positive (+) or negative (-) & \\
IIP: MA903 positive ( ) or negative $(-)$ by indirect immunoperoxidase method \\
ABC: MA903 positive (+) or negative (-) by avidin-biotin peroxidase complex method \\
WFA:Wisteria floribunda agglutinin positive ( + ) or negative (-) before neuraminidase \\
digestion \\
MDAH (MD Anderson Hospital grading sytem)
\end{tabular}

identified; the present study highlighted five weakly positive cases out of 33 examined. Although the staining noted was quite weak and focal, the implication of this finding is that alcian blue staining, which has a sensitivity of between 30 and $70 \%$, is not totally specific and thus cannot be recommended in isolation as a method to differentiate benign from malignant prostatic tissue.

Lectins are proteins or glycoproteins of nonimmune, mainly plant, origin which have highly specific binding sites for mono-or oligosaccharides. ${ }^{19}$ Changes in the saccharide composition of glycoproteins have been noted in neoplastic transformation of cells of various tissues, both on cell surfaces and in cell cytoplasms. ${ }^{20}$ In a preliminary study of prostatic lectin binding Wisteria floribunda agglutinin (WFA) binding sites were identified only in malignant prostatic tissue, but pretreatment with neuraminidase, which removes terminal sialic acid residues, revealed reactivity for WFA in both benign and malignant tissue. ${ }^{2}$ The current study again showed $90 \%$ WFA positivity in malignant tissue but also showed mainly weak reactivity for WFA in $56 \%$ of benign glands. While demonstrating excellent sensitivity, this technique lacks specificity and alone cannot be recommended for diagnostic use.

EAB 903 is a monoclonal antibody which recognises cytokeratins $1,5,10$ and 11 , corresponding to molecular weights $68,58,56.5$ and 56 kilodaltons. ${ }^{11} \mathrm{~A}$ summary of the reports on its use in the prostate to date is shown in table 4. Brawer et al examined 31 malignant cases and found no positive results. ${ }^{3}$ They described its localisation in benign glands to basal cells only, including cases of basal cell hyperplasia. Chastonay et al reported their findings in both frozen section and paraffin wax embedded material. ${ }^{7}$ The patterns of basal cell staining altered with fixation, such that frozen section material exhibited a continuous basal layer reactivity while formalin fixed tissue had an interrupted pattern. They also described the only two previous cases of positivity in malignant glands with this antibody, both in well differentiated adenocarcinomas. Nagle $e t$ $a l^{17}$ and Brawer et $a l^{18}$ examined the use of a similar, though not identical, antibody KAl in both frozen cell culture tissue and formalin fixed material; in neither instance were there any positive findings in malignant glands. Bostwick and Brawer used EAB 903 to evaluate the concept of dysplasia/prostatic intraepithelial neoplasia (PIN) and found that there was increasing disruption of the basal layer related to increasing grades of dysplasia/PIN. ${ }^{4}$ Hedrick and Epstein described three patterns of basal cell staining-continuous, continuous with focal disruption, and disrupted and applying EAB 903 to a wide variety of benign and malignant tissue, found a high incidence of disrupted basal cell staining in benign conditions and a total lack of reactivity in carcinomas. ${ }^{5}$ They cautioned against the absolute use of an absence of a basal cell layer in the diagnosis of carcinoma because of the disrupted patterns which may be seen in benign prostates. O'Malley et al described usually continuous basal cell staining in normal/hyperplastic lesions and its absence in carcinoma. ${ }^{6}$

The results of the present study show that in non-neoplastic tissue the previously described basal cell only staining is not a constant finding. The results varied with the methods used and although small numbers of cases have been examined, this gives rise to concern as to the specificity of the antibody. The dilution recommended by the manufacturers was 1 in 8000 but by a process of trial and error, the working dilution eventually chosen was 1 in 100 , as also used by Hedrick and Epstein. ${ }^{5}$ As

Table 4 Review of previous studies

\begin{tabular}{|c|c|c|c|c|c|c|}
\hline Author & Fixation & Technique & Dilution & Before treatment & Cases & No positive \\
\hline Brawer et al 1985 & FFPE & $\mathrm{ABC}$ & 1 in 2000 & Pronase & 31 & 0 \\
\hline Chastonay et al 1986 & $\begin{array}{l}\text { FFPE } \\
\text { FS }\end{array}$ & PAP & 1 in 500 & $\begin{array}{l}\text { Protease } \\
\text { Pepsin }\end{array}$ & 25 & 2 \\
\hline Nagle et al 1987 (KA1) & FS & IIP & NS & NS & 10 & 0 \\
\hline Bostwick and Brawer 1987 & FFPE & $\mathrm{ABC}$ & $\begin{array}{l}\text { sst } 1 \text { in } 2000 \\
2 \text { nd } 1 \text { in } 50\end{array}$ & Pronase & 14 & 0 \\
\hline Brawer et al 1989 (KA1) & FFPE & PAP & NS & Pronase & 37 & 0 \\
\hline Hedrick and Epstein 1989 & FFPE & $\mathrm{ABC}$ & 1 in 100 & Pronase & 58 & 0 \\
\hline O'Malley et al 1990 & FFPE & $\mathrm{ABC}$ & NS & Pepsin & 21 & 0 \\
\hline
\end{tabular}

Abbreviations:

FFPE: Formalin fixed, paraffin wax embedded material; FS: Frozen section material; ABC: Avidin-biotin-peroxidase complex method; PAP: Peroxidase anti-peroxidase method; IIP: Indirect immunoperoxidase method; NS: Not stated 
suggested for use on formalin fixed, paraffin wax embedded material, pretreatment with a protease was performed, in our case with $0 \cdot 1 \%$ crude trypsin. With the IIP method, this produced a very high carcinoma positivity rate of $36 \%(11 / 31)$, particularly in view of the findings so far in all of the studies cited of only two EAB 903 reactive carcinomas out of 200 examined. We also used the $A B C$ method as most of the other groups have done, with the exception of the KAl studies. ${ }^{718}$ This reduced the positivity rate for carcinoma considerably, and in the benign cases, unusual patterns of basal cell staining were seen as well as two totally negative results. In our hands, therefore, EAB 903 has not proved a useful adjunct to the differential diagnosis of benign from malignant lesions and indeed, the use alone, particularly with the ABC method, was more confusing than helpful.

Overall, none of the three techniques evaluated showed sufficient sensitivity and specificity to allow their recommendation for routine diagnostic use, even when evaluated as a panel of markers or by comparison with Gleason or other grading systems. Of the three methods, the simple mucin stain (AB-DPAS) provided the most useful information, although the finding of weakly positive alcianophilic material in benign glands suggests that it should not be used to the exclusion of anything else. Wisteria floribunda lectin seems to be unsuitable for further application in this context. The anticytokeratin antibody EAB 903 proved difficult in its practical use and problems arose related to the dilution and revealing method used. The ABC method would appear to be the more likely technique to yield diagnostic information but extreme caution in its interpretation is necessary in view of the "false" positive and particularly the false negative results seen in this small study. The "gold standard" in needle biopsy diagnosis still remains a good haematoxylin and eosin stained section examined by an experienced pathologist.
1 Pinder SE, McMahon RFT. Mucins in prostatic carcinoma. Histopathology 1990;16:43-6.

2 Rhodes H, McMahon RFT, McWilliam LJ, Stoddart RW. Lectin histochemistry of human prostatic gland - normal, hyperplastic and carcinomatous. $\mathcal{f}$ Pathol 1990 161:359A.

3 Brawer MK, Peehl DM, Stamey TA, Bostwick DG. Keratin immunoraeactivity in the benign and neoplastic human prostate. Cancer Res 1985;45:3663-7.

4 Bostwick DG, Brawer MK. Prostatic intra-epithelial neoplasia and early invasion in prostate cancer. Cancer 1987;59:788-94.

5 Hedrick L, Epstein JI. Use of keratin 903 as an adjunct in the diagnosis of prostatic carcinoma. Am $\mathcal{f}$ Surg Pathol 989;13:389-96.

6 O'Malley FP, Grignon DJ, Shum DT. Usefulness of immunoperoxidase staining with high-molecular-weight cytokeratin in the differential diagnosis of small-acinar lesions of the prostate gland. Virchows Arch (Pathol Anat) 1990;417:191-6.

7 Chastonay P, Hurlimann J, Gardiol D. Biological tissue markers in benign and malignant disease of the hum

prostate. Virchows Arch (Pathol Anat) 1986;410:221-9.
8 Tannenbaum M, Romas N. The prostate gland. In: Silverberg, SG, ed. Principles and practice of surgical pathology berg, SG, ed. Principles and practice of surgical pathology.
Vol 2. New York: John Wiley and Sons, 1983;1189-217.

9 Gleason DF. Histologic grading and clinical staging of carcinoma of the prostate. In: Tannenbaum $M$, ed. Urologic Pathology: The prostate. Philadelphia: Lea and Febiger, 1977:171-98.

10 Brawn PN, Ayala AG, von Eschenback AC, Hussey DH, Johnson DE. Histologic grading study of prostate adenocarcinoma: The development of a new system and comparison with other methods-A preliminary study. Cancer 1982;49:525-32.

11 Gown AM, Vogel AM. Monoclonal antibodies to human intermediate filaments II. Distribution of filament pro-
teins in normal human tissues. Am $\mathcal{f}$ Pathol 1984; 114:309-21.

12 Elbadawi A, Craig W, Linke CA, Cooper RA. Prostatic mucinous carcinoma. Urology 1979;13:658-66.

13 Franks LM, O'Shea JD, Thomson AER. Mucin in the prostate: a histochemical study in normal glands, latent, clinical and colloid cancers. Cancer 1964;17:983-91.

14 Hukill PB, Vidone RA. Histochemistry of mucus and other polysaccharides in tumors. II. Carcinoma of the prostate. Lab Invest 1967;16:395-406.

15 Ro JY, Grignon DJ, Troncoso P, Ayala AG. Mucin in prostatic carcinoma. Semin Diagnost Pathol 1988;5: 273-83.

16 Abel PD, Keane P, Leathem A, Tebbutt S, Williams G. Change in glycoconjugate for the binding site of the lectin Change in glycoconjugate for the binding site of the lectin Ulex europeus 1 following malignant transform
prostatic epithelium. $B r \mathcal{Y}$ Urol 1989;63:183-5.

17 Nagle RB, Ahmann FR, McDaniel KM, Paquin ML, Clark VA, Celniker A. Cytokeratin characterization of human prostatic carcinoma and its derived cell lines. Cancer Res 1987;47:281-6.

18 Brawer MK, Nagle RB, Pitts W, Freiha F, Gamble SL Keratin immunoreactivity as an aid to the diagnosis of persistent adenocarcinomas in irradiated human prostates. Cancer 1989;63:454-60.

19 Damjanov I. Lectin cytochemistry and histochemistry. Lab Invest 1987;57:5-20.

20 Cooper HS. Lectins as probes in histochemistry and immunohistochemistry-the peanut (Arachis hypogaea) lectin. Hum Pathol 1984;15:904-6. 